\title{
Community structure, biomass and productivity of epilithic algal communities on the Great Barrier Reef: dynamics at different spatial scales*
}

\author{
D. W. Klumpp, A. D. McKinnon \\ Australian Institute of Marine Science, PMB 3, Townsville MC, Queensland 4810, Australia
}

\begin{abstract}
Epilithic algal communities (EAC) covered a high proportion of the reef flats (50 to $80 \%$ ) and reef slopes ( 30 to $70 \%$ ) on coral reefs of the the north, central and southern regions of the Great Barrier Reef (GBR). Crustose coralline algae and turf algae (fine and damselfish territory types) dominated the EAC in reef flat habitats, except in the nearshore region where turf algae predominated. Turfs also dominated the EAC on reef slopes. Patches of crustose coralline algae had a higher biomass, but a lower photosynthetic rate than the equivalent area of fine turf algae. The net result was that these 2 main forms of epilithic algae had comparable rates of areal productivity. The productivity of turfdominated communities was inversely correlated with algal biomass. Epilithic algal communities from various reef habitats at the same depth had equal areal and biomass-specific productivity, regardless of their location on transects extending both across and also along the GBR. EAC productivity changed in a predictable manner with season (maximum in summer, minimum in winter) and depth (decreasing with depth). Photoadaptation by the EAC was observed (variable $I_{k}$, photosynthetic efficiency, and $\left.P_{\max }\right)$, and this served to minimize the effect of changes in irradiance on the productivity of these communities. The EAC at $10 \mathrm{~m}$ on reef slopes had approximately half the areal productivity of the community on the adjacent reef flat, but the EAC from these habitats had a similar biomass-specific productivity. Productivity of the EAC per unit area of reef, which takes into account the rugosity and coverage of reefs by the EAC in particular habitats, varied between reefs, and ranged from $150 \mathrm{~g} \mathrm{C} \mathrm{m}^{-2}$ $\mathrm{yr}^{-1}$ on the reef flat of the nearshore reef and on all reef slopes, to $500 \mathrm{~g} \mathrm{C} \mathrm{m}^{-2} \mathrm{yr}^{-1}$ on some mid-and outer-shelf reef flats. There was no apparent latitudinal pattern of change in EAC productivity per unit area of reef. Thus, availability of the EAC, the major food resource of grazers on coral reefs, appears to correlate well with known large-scale variations in grazing activity.
\end{abstract}

\section{INTRODUCTION}

The epilithic algal community (EAC) of coral reefs is a diverse assemblage of crustose coralline and turf algae growing upon coral rock. In this case, the term 'turf' (sensu Dahl 1974, Wanders 1977 and Borowitzka et al. 1978) refers to the multispecific and inconspicuous association of unicellular, and short (usually $<1 \mathrm{~cm}$ high), simple filamentous algae, and should not be confused with the more complex and fleshy forms of 'turfs', such as that studied by Hay (1981). Usually, all major algal phyla are represented in the $\mathrm{EAC}$, but

\footnotetext{
- AIMS Contribution No. 647
}

Chlorophytes and Rhodophytes tend to dominate (Scott \& Russ 1987, Hackney et al. 1989, Klumpp \& Polunin 1989).

For a number of reasons, the EAC is thought to play an extremely important role in the trophodynamics of coral reefs. Firstly, a large proportion of the net primary production within specific habitats of coral reefs is provided by the EAC (Odum \& Odum 1955, Marsh 1976. Brawley \& Adey 1977. Borowitzka et al. 1983, Klumpp \& McKinnon 1989). This is most clearly evident for the shallow habitats, where it has been relatively easy to measure the metabolic rates of benthic reef communities. However, relatively little is known about the productivity of benthic plant communities on reef slopes and in deep lagoons. Epilithic algae are 
intensively grazed (Hatcher 1983, Carpenter 1986, Russ 1987, Klumpp \& Polunin 1989), and in the process are maintained in a state of low biomass, but rapid turnover (Rogers \& Salesky 1981, Carpenter 1985, Klumpp et. al. 1987). In the few cases where whole reef systems have been examined, the biomass of the EAC is found to be considerable due to its extensive coverage of reef surfaces (up to $80 \%$ in some habitats), and the high rugosity of the reef substratum. Hence, the EAC almost certainly makes a substantial contribution to the areal productivity of most reefs. Moreover, a high proportion of the carbon produced by the coral reef EAC is thought to be directly available to the reef food web via herbivorous grazers. Indeed, recent studies of reef flats have demonstrated that grazers consume around half of the annual net production of the EAC (Hatcher 1983, Klumpp \& Polunin 1990), with the balance presumably channelled into detrital pathways in the form of exudates and particulate matter.

The main goal of this study was to examine the natural (in situ) variations in community structure, biomass, photosynthesis-irradiance (P-I) relationship and primary productivity of the EAC on the Great Barrier Reef (GBR). Comparisons are made between seasons, successive years, and different habitats and reefs situated across and along the continental shelf. This is an extension of a study which focussed on the differences between habitats and seasons within a single reef (Davies Reef, central GBR i see Klumpp \& McKinnon 1989). Data on primary production of the EAC are compared with the rugosity and nature of the reef substratum to estimate the contribution made by the EAC to whole reef productivity.

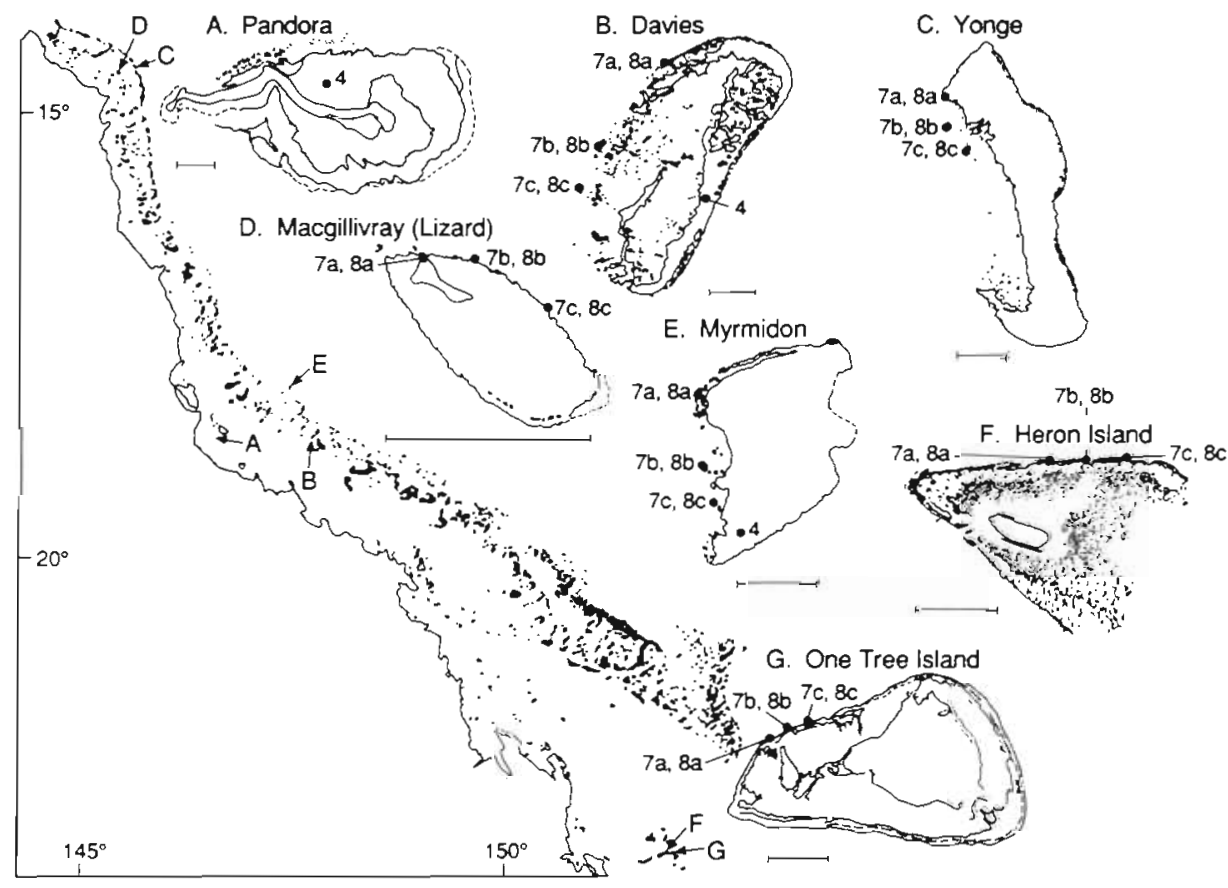

\section{MATERIALS AND METHODS}

Study area and sampling techniques. This study was carned out along 2 major transects designed to give a wide geographical coverage of the Great Barrier Reef (GBR, Fig. 1). The first of these was a central GBR cross-shelf transect comprising a reef on the inner(Pandora), mid- (Davies) and outer-shelf (Myrmidon). General environmental and structural features of these reefs are described elsewhere (Done 1982, Bradbury et al. 1986). The second transect comprised a pair of reefs on the mid-and outer-shelf at 3 latitudes (Fig. 1); McGillivray and Yonge (northern, $15^{\circ} \mathrm{S}$ ), Davies and Myrmidon (central, $19^{\circ}$ S), and Heron and One Tree Islands (southern, $25^{\circ} \mathrm{S}$ ).

Experimental plates (ca $8 \times 8 \times 2 \mathrm{~cm}$ ) cut from the coral Porites spp. were bolted directly to the reef substratum in a haphazard manner within different reef zones (zonal nomenclature of Klumpp \& McKinnon 1989). Within each zone there were 3 sites, each being ca $9 \mathrm{~m}^{2}$ and $30 \mathrm{~m}$ apart. At Davies and Myrmidon Reefs, 3 zones were studied; the middle of the windward reef flat (Zone 4), the crest of the leeward reef flat (Zone 7), and on the slope adjacent to Zone 7 at a depth of $10 \mathrm{~m}$ (Zone 8 ). The simple structure and shallow nature of the adjacent slope on Pandora Reef meant that it provided only 1 comparable location (Zone 4/7). The latitudinal study was restricted to Zones 7 and 8 on each of the 6 reefs, with both zones replicated at 3 sites (labelled a, b, c in Fig. 1), approximately $500 \mathrm{~m}$ apart. Plates were left in the field for 6 to 12 mo to establish a 'natural' epilithic algal community (EAC; see Klumpp \& McKinnon 1989).

Fig. 1. Location of sampling sites on 7 reefs of the Great Barrier Reel, Australia. Habitats sampled within reefs were the mid-reef flat (Zone 4), back-reef crest (Zone 7) and reef slope (Zone 8); (a), (b), (c): replicate sites of Zones $7 \& 8$ on 6 reefs. Scale bars: A: $100 \mathrm{~m}$; $B$ to $\mathrm{G}: 1 \mathrm{~km}$ 
Community structure, biomass and metabolism of EAC on plates. Coral plates were sampled for measurement of their algal community structure, biomass and productivity in August (winter) and October (spring) 1986, and February (summer) and May (autumn) 1987 on the cross-shelf reefs, and from the latitudinal reefs in February 1988 and July-August 1988. Generally, this involved measurements on 4 plates from each site, amounting to 12 per zone, on each occasion. Measurements of algal productivity, by $24 \mathrm{~h}$ deployments of 3 in situ respirometers, could be made on 12 separate plates at a time, and these were conducted such that different zones on a particular reef were compared simultaneously. Measurements continued on consecutive days until 12 plates had been measured from each reef zone. Details on terminology, definitions and methodology for sample preparation; measurement of algal community structure (according to functional forms); biomass; and metabolism (dark respiration rate, photosynthesis-irradiance (P-I) relationships, diel productivity); and data analysis are given in Klumpp et al. (1987) and Klumpp \& McKinnon (1989), and therefore are only summarized here. The undersurface of coral plates were scraped in situ and transferred to the Perspex respirometer chambers. During the incubation the water in each chamber was stirred continuously, and the water exchanged completely every $15 \mathrm{~min} . \mathrm{O}_{2}$ concentration, temperature and irradiance were recorded simultaneously at $1 \mathrm{~min}$ intervals. P-I relationships were modelled using the hyperbolic tangent function. Daily production and consumption of $\mathrm{O}_{2}$ by algae were calculated from continuous 24 h records of $\mathrm{O}_{2}$ flux, and converted to $\mathrm{C}$ flux assuming that $\mathrm{PQ}=\mathrm{RQ}=1\left(1 \mathrm{~g} \mathrm{O}_{2}=0.375 \mathrm{~g} \mathrm{C}\right)$. Immediately after respirometry, the percent cover of the EAC on the entire upper surface of each plate was estimated visually and the algae recorded in 4 categories: fine turf ( $<1 \mathrm{~mm}$ high), damselfish-territory turf, crustose coralline, and encrusting brown. Plates were dried, measured for surface area, and the EAC removed by scraping until the underlying coral matrix was exposed. Coral scrapings were analysed for total organic $\mathrm{C}$ as the measure of EAC biomass.

Irregularity and community structure of reef surfaces. The type and irregularity of the reef surface, at the sites where algal production was measured, were surveyed at Pandora, Myrmidon, Heron and One Tree Reefs in October 1989, and at MacGillivray and Yonge Reefs in December 1989. Reef surface type, expressed as proportional coverage by sand, and 7 functional groups of biota (as defined in Klumpp et al. 1987): fine turf, damselfish-territory turf, crustose coralline algae, encrusting brown algae, macroalgae (e.g. Halimeda spp.), hard corals and other fauna (e.g. soft corals, sponges), was quantified using line transects (method- ology from Mundy 1990). Four replicate transects of $30 \mathrm{~m}$ each were surveyed at each site (i.e. 12 transects per zone) for all reefs except Heron Is. and One Tree Is., which had 2 replicates per site (i.e. 6 transects per zone). Data for Davies Reef was derived from a previous study (Klumpp et al. 1987).

The topographic surface area of the reef at each of the above survey sites (10 replicates per site, which from our experience accounted for most variance), taking into account surface irregularities, was estimated using chain ( $1 \mathrm{~cm}$ links) transects. This involved running a length of chain (length $C=3 \mathrm{~m}$ ) in a straight line across the reef surface contour, then measuring the direct distance $(H)$ between the chain ends. From this a surface irregularity factor (IF) was calculated as the ratio of $C$ to $H$. Topographic area, which was used to calculate EAC production per unit area of reef surface, was derived from the product of $(I F)^{2}$ and planar area.

Data analysis. Variation in algal community structure, biomass, and metabolic parameters were examined in relation to zone, season and reef location using ANOVA. Where residual analysis indicated significantly heterogeneous variances, the data were logtransformed to make the variance homogeneous. Where differences were significant, means were compared using the Student-Newman-Keuls (SNK) procedure. Data analysis was carried out as described by Klumpp \& McKinnon (1989).

The effects of algal composition (using percent crustose coralline cover as an index) on biomass, and net productivity in terms of both biomass and area were estimated by regression and ANCOVA. Substrata with $>50 \%$ cover of damselfish turfs were excluded from statistical analyses because these turfs were known to have twice the biomass and production of other types of EAC (for details see Klumpp \& McKinnon 1989). All statistical analyses were performed using programs from SAS (1985)

\section{RESULTS}

\section{Structure of the EAC on coral plates}

The percent cover of coral plates by the 4 major functional components of the epilithic algal community (fine turfs, damselfish territory turf, crustose coralline algae, and encrusting brown algae; descriptions in Klumpp \& McKinnon 1989), averaged over all seasons, was similar for the 2 reef-flat zones ( $4 \& 7$ ) on Davies and Myrmidon Reefs (Fig. 2). Both turfs and coralline algae were important in these habitats, but coralline algae were more abundant on reef crests (Zone 7 ). Fine turf dominated (57 to $67 \%$ cover) and coralline 


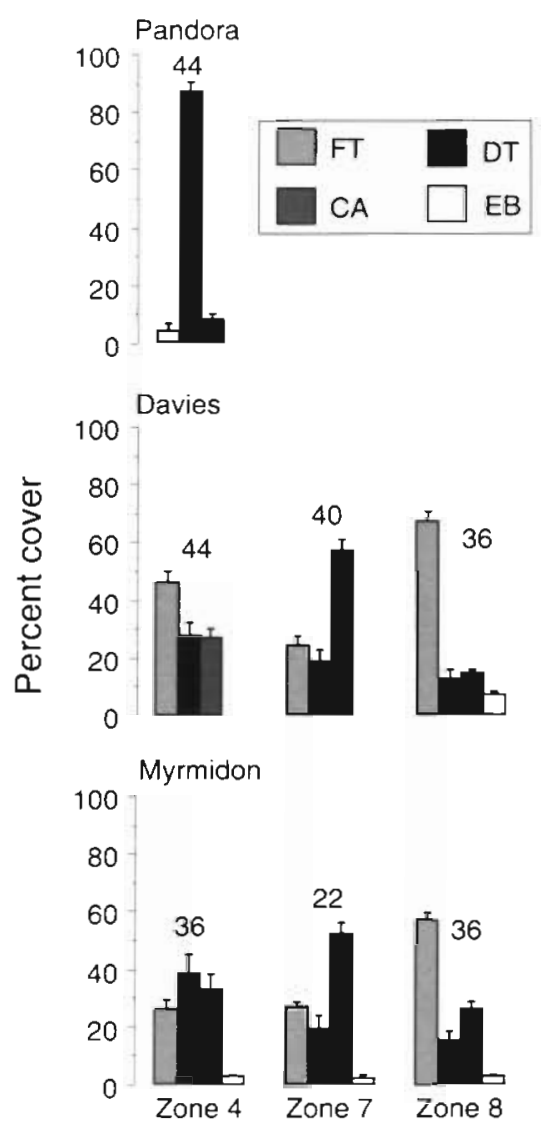

Fig. 2. Composition (\% cover) of the EAC for each of 3 zones (see Fig. 1) sampled during 1986 to 1987 on the 3 reefs of the cross-shelf transect. Data are means + SE percent cover; $n$ values are above bars. FT; fine turf; DT: damselfish territory turf $_{i} \mathrm{CA}$ : crustose coralline algae; EB: encrusting browns

algae were much less abundant (ca $20 \%$ cover) in the reef-slope algal community. The reef flat on Pandora Reef on the inner-shelf differed markedly from similar habitats on the mid- and outer-shelf reefs in that coralline algae were rare, and $87 \%$ of algae was damselfish territory turf. EAC structure did not vary significantly with season ( $F$-test, $p>0.05$ ).

The percent cover of algal community components within zones of adjacent reefs along the latitudinal transect was similar, and was generally so between all transect reefs (Fig. 3). As noted for the cross-shelf transect, coralline algae dominated at Zone 7 , especially on the high latitude reefs (Heron and One Tree Islands), and these were replaced by fine turfs on all reef slopes (Zone 8).

\section{EAC biomass}

Algal biomass did not differ significantly across the shallow zones of Davies and Myrmidon Reefs (Fig. 4), but on both reefs it varied seasonally (F-test, $\mathrm{p}<0.001$ ), tending to be lowest in summer (February) and highest in winter-spring (August to October). On Pandora Reef this pattern was reversed (Fig. 4). Otherwise, the EAC of all 3 reef flats exhibited a similar seasonal range in biomass (max/min ca 1.4). Reef slopes by comparison were characterised by a seasonally stable, and significantly lower algal community biomass, which was only 40 to $60 \%$ of that on the adjacent flat.

Seasonal and zonal patterns in EAC biomass for the 6 reefs on the latitudinal transect (Fig. 5) were similar to those observed on the cross-shelf transect. Algal biomass on Davies and Myrmidon reefs did not change significantly between 1987 and 1988. In addition, during winter, algal biomass on reef flats (Zone 7 ) tended to increase with increasing latitude ( $F$-test, $\mathrm{p}=0.0003$; SNK test), but no significant differences occurred between reefs in summer. Thus, the seasonal range in biomass was greatest on the high latitude reefs and least on reefs in the far north (Fig. 5), despite most reef flats sampled having significantly higher biomass in winter than summer. On reef slopes, algal biomass did not vary significantly between reefs or seasons.

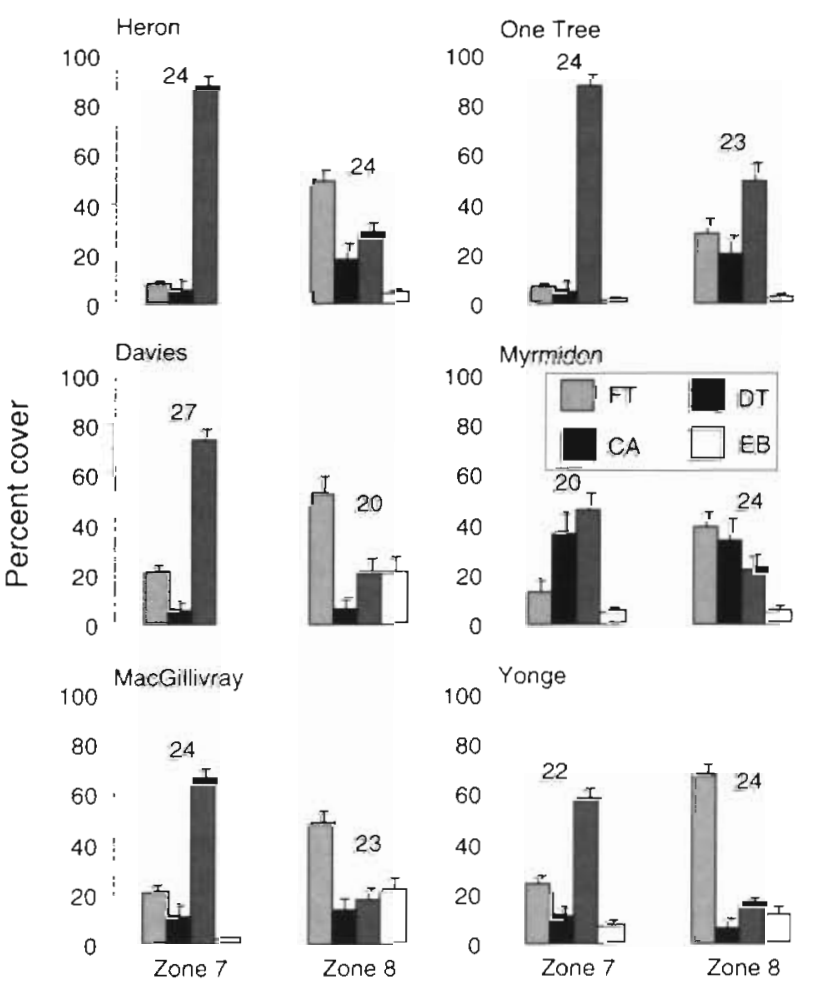

Fig. 3. Composition ( $\%$ cover) of the EAC for each of 2 zones (see Fig. 1) sampled during 1988 on the 6 reefs of the latitudinal transect. Data are means + SE percent cover; n-values are above bars. Algal type codes as in Fig. 2 

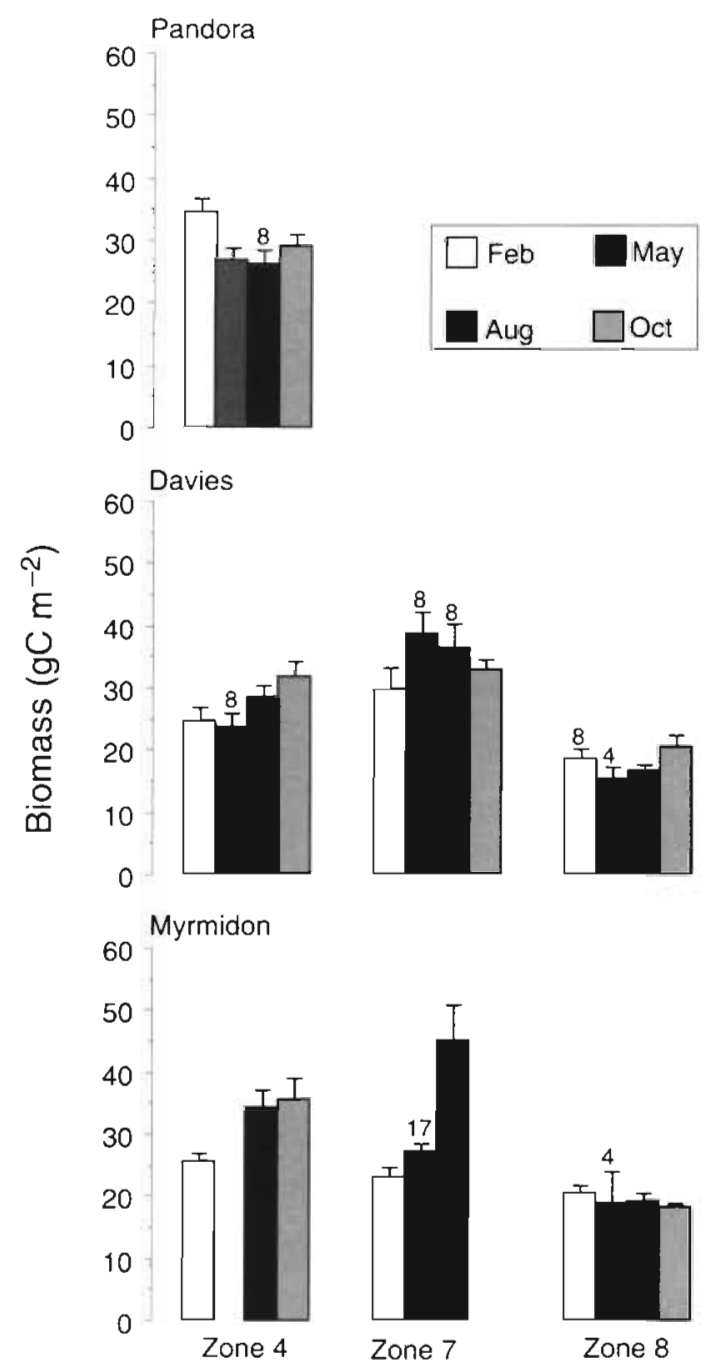

Fig. 4. Spatial and temporat variation in the biomass $\left(\mathrm{g} \mathrm{C} \mathrm{m}^{-2}\right)$ of EAC on the reefs of the cross-shelf transect. Data are means $+\mathrm{SE}_{;} \mathrm{n}=12$, or as given above bars

\section{Photosynthesis-irradiance relationships}

The net photosynthetic rate $(P)$ of the EAC on cloudless days was symmetrical about the corresponding midday peak in irradiance $(I)$ for all seasons, zones and reefs sampled. This symmetry, as previously illustrated for the EAC on Davies Reef (Klumpp \& McKinnon 1989), indicates a lack of photoinhibition.

The maximum net photosynthetic rate $\left(P_{\max }\right)$ of the EAC, in both areal and biomass-specific terms, varied seasonally, with the maxima in summer and the minima in winter in all zones, on all reefs (Tables $1 \& 2$ ). Generally, areal $P_{\max }$ was higher on reef flats than on slopes, but biomass-specific $P_{\max }$ was highest on reef slopes in all seasons. There were few clear cross-shelf or latitudinal patterns to the inter-reef variations in

$P_{\max }$. In winter, reef location along the latitudinal transect had a significant effect on $P_{\max }$ of the EAC from both the reef flat and slope habitats (F-test, $\mathrm{p}<0.001$ ), although only the reef flat provided a clear pattern of a consistent decline in biomass-specific $P_{\max }$ with increasing latitude (SNK test). No significant differences in the photosynthetic rate of the EAC were found between reefs in summer ( $F$-test).

The initial slope of the P-I curve $(\alpha)$, and the irradiance at the point where $\alpha$ intersects with the line delimited by $P_{\max }\left(I_{\mathrm{k}}\right)$, and the way in which these 2 parameters change in response to changes in irradiance are useful indicators of photoadaptation in plants. $I_{\mathrm{k}}$ was found to vary seasonally, increasing from winter to summer in all habitats and on all reefs (Tables $1 \& 2$ ). Invariably, $I_{k}$ of the EAC in shallow reef-flat habitats was higher than that of the reef slope communities. Although the $I_{\mathrm{k}}$ values of some reefs differed significantly (e.g. reef flats in summer: Heron Island > One Tree Island), there was no clear spatial pattern to these differences.
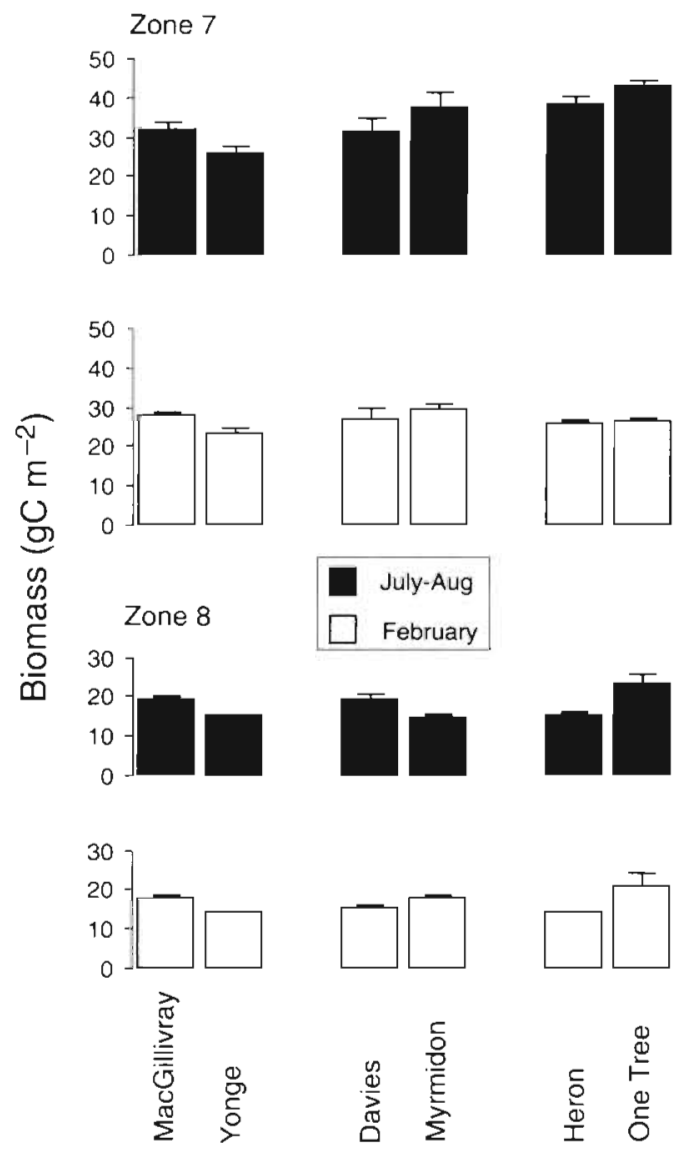

Fig. 5. Spatial and temporal variation in the biomass $\left(\mathrm{g} \mathrm{C} \mathrm{m}^{-2}\right)$ of $\mathrm{EAC}$ on the reefs of the latitudinal transect. Data are means $+\mathrm{SE} ; \mathrm{n}=12$ 
Table 1. Cross-shelf variation in photosynthesis-irradiance parameters standardised to area, $\left(\mathrm{a}_{;} \mu \mathrm{g} \mathrm{O}_{2} \mathrm{~h}^{-1} \mathrm{~cm}^{-2}\right)$ and to biomass (b; $\mu \mathrm{g} \mathrm{O}_{2} \mathrm{~h}^{-1} \mathrm{mg} \mathrm{C}^{-1}$ ), 1986-1987. Data are means $\pm \mathrm{SE}$

\begin{tabular}{|c|c|c|c|c|c|c|c|c|c|c|}
\hline \multirow[t]{2}{*}{ Zone } & \multirow[t]{2}{*}{ Month } & \multirow[t]{2}{*}{$\mathrm{n}$} & \multicolumn{2}{|c|}{$P_{\max }$} & \multicolumn{2}{|c|}{$R$} & \multicolumn{2}{|c|}{$\alpha\left(=P_{\max } / I_{k}\right)$} & \multirow{2}{*}{\multicolumn{2}{|c|}{$\begin{array}{l}I_{\mathrm{k}} \\
\left(\mu \mathrm{E} \mathrm{m}^{-2} \mathrm{~s}^{-1}\right)\end{array}$}} \\
\hline & & & a & $\mathrm{b}$ & $\mathrm{a}$ & $\mathrm{b}$ & $\mathrm{a}$ & $b$ & & \\
\hline \multicolumn{11}{|c|}{ Pandora Reef } \\
\hline \multirow[t]{4}{*}{4} & Aug & 7 & $\begin{array}{r}56.3 \\
\pm 2.5\end{array}$ & $\begin{array}{c}22.3 \\
\pm 2.4\end{array}$ & $\begin{array}{c}8.6 \\
\pm 0.5\end{array}$ & $\begin{array}{c}3.4 \\
\pm 1.0\end{array}$ & $\begin{array}{c}0.17 \\
\pm 0.01\end{array}$ & $\begin{array}{c}0.07 \\
\pm 0.01\end{array}$ & $\begin{array}{c}327 \\
\pm 13\end{array}$ & $\begin{array}{c}51 \\
\pm 2\end{array}$ \\
\hline & Oct & 12 & $\begin{array}{r}80.9 \\
\pm 3.7\end{array}$ & $\begin{array}{r}28.4 \\
\pm 1.2\end{array}$ & $\begin{array}{r}11.8 \\
\pm 0.8\end{array}$ & $\begin{array}{c}4.1 \\
\pm 0.2\end{array}$ & $\begin{array}{c}0.19 \\
\pm 0.01\end{array}$ & $\begin{array}{c}0.07 \\
\pm 0\end{array}$ & $\begin{array}{l}429 \\
\pm 9\end{array}$ & $\begin{array}{r}65 \\
\pm 2\end{array}$ \\
\hline & Feb & 8 & $\begin{array}{r}91.1 \\
\pm 5.3\end{array}$ & $\begin{array}{r}26.9 \\
\pm 2.3\end{array}$ & $\begin{array}{r}14.2 \\
\pm 0.7\end{array}$ & $\begin{array}{c}4.2 \\
\pm 0.4\end{array}$ & $\begin{array}{c}0.18 \\
\pm 0.01\end{array}$ & $\begin{array}{c}0.05 \\
\pm 0\end{array}$ & $\begin{array}{l}517 \\
\pm 19\end{array}$ & $\begin{array}{l}82 \\
\pm 3\end{array}$ \\
\hline & May & 11 & $\begin{array}{r}67.7 \\
\pm 1.8\end{array}$ & $\begin{array}{c}26.4 \\
\pm 1.8\end{array}$ & $\begin{array}{c}8.8 \\
\pm 0.3\end{array}$ & $\begin{array}{c}3.5 \\
\pm 0.3\end{array}$ & $\begin{array}{c}0.17 \\
\pm 0.01\end{array}$ & $\begin{array}{c}0.07 \\
\pm 0\end{array}$ & $\begin{array}{c}404 \\
\pm 15\end{array}$ & $\begin{array}{r}55 \\
\pm 2\end{array}$ \\
\hline \multicolumn{11}{|c|}{ Davies Reef } \\
\hline \multirow[t]{4}{*}{4} & Aug & 12 & $\begin{array}{r}42.1 \\
\pm 3.6\end{array}$ & $\begin{array}{r}16.2 \\
\pm 2.1\end{array}$ & $\begin{array}{c}7.4 \\
\pm 0.6\end{array}$ & $\begin{array}{c}2.8 \\
\pm 0.3\end{array}$ & $\begin{array}{c}0.17 \\
\pm 0.02\end{array}$ & $\begin{array}{c}0.07 \\
\pm 0.01\end{array}$ & $\begin{array}{r}270 \\
\pm 32\end{array}$ & $\begin{array}{l}46 \\
\pm 3\end{array}$ \\
\hline & Oct & 12 & $\begin{array}{r}59.0 \\
\pm 5.1\end{array}$ & $\begin{array}{r}19.6 \\
\pm 2.1\end{array}$ & $\begin{array}{c}9.8 \\
\pm 0.7\end{array}$ & $\begin{array}{c}3.2 \\
\pm 0.3\end{array}$ & $\begin{array}{c}0.17 \\
\pm 0.01\end{array}$ & $\begin{array}{c}0.06 \\
\pm 0.01\end{array}$ & $\begin{array}{c}348 \\
\pm 16\end{array}$ & $\begin{array}{l}61 \\
\pm 2\end{array}$ \\
\hline & Feb & 12 & $\begin{array}{l}66.1 \\
\pm 2.4\end{array}$ & $\begin{array}{l}29.1 \\
\pm 2.2\end{array}$ & $\begin{array}{r}12.6 \\
\pm 0.5\end{array}$ & $\begin{array}{c}5.5 \\
\pm 0.4\end{array}$ & $\begin{array}{c}0.18 \\
\pm 0.01\end{array}$ & $\begin{array}{c}0.08 \\
\pm 0.01\end{array}$ & $\begin{array}{r}375 \\
\pm 17\end{array}$ & $\begin{array}{l}74 \\
\pm 3\end{array}$ \\
\hline & May & 8 & $\begin{array}{r}69.9 \\
\pm 6.2\end{array}$ & $\begin{array}{r}31.5 \\
\pm 3.7\end{array}$ & $\begin{array}{c}9.2 \\
\pm 1.1\end{array}$ & $\begin{array}{r}4.2 \\
\pm 0.6\end{array}$ & $\begin{array}{c}0.18 \\
\pm 0.02\end{array}$ & $\begin{array}{c}0.09 \\
+0.02\end{array}$ & $\begin{array}{c}387 \\
\pm 24\end{array}$ & $\begin{array}{l}58 \\
\pm 3\end{array}$ \\
\hline \multirow[t]{4}{*}{7} & Aug & 8 & $\begin{array}{r}51.9 \\
\pm 2.4\end{array}$ & $\begin{array}{r}15.3 \\
\pm 1.5\end{array}$ & $\begin{array}{c}8.7 \\
\pm 0.4\end{array}$ & $\begin{array}{c}2.6 \\
\pm 0.3\end{array}$ & $\begin{array}{c}0.17 \\
\pm 0.01\end{array}$ & $\begin{array}{c}0.05 \\
\pm 0\end{array}$ & $\begin{array}{r}315 \\
\pm 12\end{array}$ & $\begin{array}{l}54 \\
\pm 2\end{array}$ \\
\hline & Oct & 9 & $\begin{array}{r}55.0 \\
\pm 3.2\end{array}$ & $\begin{array}{r}17.0 \\
\pm 1.6\end{array}$ & $\begin{array}{r}10.1 \\
\pm 0.7\end{array}$ & $\begin{array}{c}3.1 \\
\pm 0.3\end{array}$ & $\begin{array}{c}0.15 \\
\pm 0.01\end{array}$ & $\begin{array}{c}0.05 \\
\pm 0.01\end{array}$ & $\begin{array}{r}401 \\
+41\end{array}$ & $\begin{array}{l}74 \\
\pm 5\end{array}$ \\
\hline & Feb & 12 & $\begin{array}{r}71.8 \\
\pm 5.0\end{array}$ & $\begin{array}{l}26.2 \\
\pm 2.0\end{array}$ & $\begin{array}{r}12.5 \\
\pm 0.9\end{array}$ & $\begin{array}{c}4.5 \\
\pm 0.3\end{array}$ & $\begin{array}{c}0.17 \\
\pm 0.01\end{array}$ & $\begin{array}{c}0.06 \\
\pm 0.01\end{array}$ & $\begin{array}{r}414 \\
+16\end{array}$ & $\begin{array}{l}74 \\
\pm 3\end{array}$ \\
\hline & May & 8 & $\begin{array}{r}57.2 \\
\pm 4.3\end{array}$ & $\begin{array}{r}15.6 \\
\pm 1.9\end{array}$ & $\begin{array}{l}9.3 \\
\pm 0.6\end{array}$ & $\begin{array}{c}2.5 \\
\pm 0.3\end{array}$ & $\begin{array}{c}0.18 \\
\pm 0.02\end{array}$ & $\begin{array}{c}0.05 \\
\pm 0.01\end{array}$ & $\begin{array}{r}328 \\
\pm 18\end{array}$ & $\begin{array}{r}56 \\
\pm 3\end{array}$ \\
\hline \multirow[t]{4}{*}{8} & Aug & 11 & $\begin{array}{r}36.9 \\
\pm 1.0\end{array}$ & $\begin{array}{r}23.8 \\
\pm 2.1\end{array}$ & $\begin{array}{c}6.3 \\
\pm 0.2\end{array}$ & $\begin{array}{c}4.0 \\
\pm 0.4\end{array}$ & $\begin{array}{c}0.20 \\
\pm 0.01\end{array}$ & $\begin{array}{c}0.12 \\
\pm 0.01\end{array}$ & $\begin{array}{r}195 \\
\pm 12\end{array}$ & $\begin{array}{l}34 \\
\pm 2\end{array}$ \\
\hline & Oct & 12 & $\begin{array}{r}45.4 \\
\pm 1.6\end{array}$ & $\begin{array}{r}23.6 \\
\pm 1.8\end{array}$ & $\begin{array}{l}8.7 \\
+0.3\end{array}$ & $\begin{array}{l}4.5 \\
\pm 0.3\end{array}$ & $\begin{array}{c}0.20 \\
\pm 0.01\end{array}$ & $\begin{array}{c}0.10 \\
\pm 0.01\end{array}$ & $\begin{array}{l}228 \\
\pm 8\end{array}$ & $\begin{array}{r}45 \\
\pm 2\end{array}$ \\
\hline & Feb & 8 & $\begin{array}{r}65.7 \\
\pm 3.1\end{array}$ & $\begin{array}{c}37.1 \\
\pm 2.5\end{array}$ & $\begin{array}{r}11.2 \\
\pm 0.4\end{array}$ & $\begin{array}{c}6.3 \\
\pm 0.4\end{array}$ & $\begin{array}{c}0.29 \\
\pm 0.01\end{array}$ & $\begin{array}{c}0.16 \\
\pm 0.01\end{array}$ & $\begin{array}{l}229 \\
\pm 8\end{array}$ & $\begin{array}{r}40 \\
\pm 1\end{array}$ \\
\hline & May & 4 & $\begin{array}{r}38.8 \\
\pm 1.6\end{array}$ & $\begin{array}{r}26.7 \\
\pm 3.3\end{array}$ & $\begin{array}{c}6.4 \\
\pm 0.1\end{array}$ & $\begin{array}{r}4.4 \\
+0.6\end{array}$ & $\begin{array}{c}0.24 \\
\pm 0.02\end{array}$ & $\begin{array}{c}0.16 \\
\pm 0.02\end{array}$ & $\begin{array}{r}165 \\
\pm 12\end{array}$ & $\begin{array}{r}28 \\
\pm 2\end{array}$ \\
\hline \multicolumn{11}{|c|}{ Myrmidon Reef } \\
\hline \multirow[t]{3}{*}{4} & Aug & 12 & $\begin{array}{r}53.1 \\
\pm 3.6\end{array}$ & $\begin{array}{r}16.7 \\
\pm 1.8\end{array}$ & $\begin{array}{c}9.4 \\
\pm 0.5\end{array}$ & $\begin{array}{c}3.0 \\
\pm 0.3\end{array}$ & $\begin{array}{c}0.17 \\
\pm 0.02\end{array}$ & $\begin{array}{c}0.05 \\
\pm 0.01\end{array}$ & $\begin{array}{r}326 \\
\pm 21\end{array}$ & $\begin{array}{r}62 \\
\pm 5\end{array}$ \\
\hline & Oct & 12 & $\begin{array}{c}66.1 \\
\pm 11.0\end{array}$ & $\begin{array}{c}27.2 \\
\pm 11.6\end{array}$ & $\begin{array}{r}10.8 \\
\pm 0.8\end{array}$ & $\begin{array}{l}3.9 \\
\pm 1.0\end{array}$ & $\begin{array}{c}0.14 \\
\pm 0.02\end{array}$ & $\begin{array}{l}0.05 \\
\pm 0.02\end{array}$ & $\begin{array}{r}476 \\
\pm 35\end{array}$ & $\begin{array}{l}83 \\
\pm 3\end{array}$ \\
\hline & Feb & 12 & $\begin{array}{r}116.9 \\
\pm 10.8\end{array}$ & $\begin{array}{l}46.6 \\
\pm 4.4\end{array}$ & $\begin{array}{r}19.6 \\
\pm 1.5\end{array}$ & $\begin{array}{c}7.8 \\
\pm 0.6\end{array}$ & $\begin{array}{c}0.26 \\
\pm 0.02\end{array}$ & $\begin{array}{c}0.10 \\
\pm 0.01\end{array}$ & $\begin{array}{r}452 \\
\pm 20\end{array}$ & $\begin{array}{r}79 \\
\pm 3\end{array}$ \\
\hline 7 & Aug & 11 & $\begin{array}{r}66.4 \\
\pm 7.6\end{array}$ & $\begin{array}{l}20.1 \\
\pm 6.2\end{array}$ & $\begin{array}{r}11.4 \\
\pm 1.0\end{array}$ & $\begin{array}{l}3.3 \\
\pm 0.9\end{array}$ & $\begin{array}{c}0.14 \\
\pm 0.01\end{array}$ & $\begin{array}{l}0.04 \\
\pm 0.01\end{array}$ & $\begin{array}{r}459 \\
\pm 21\end{array}$ & $\begin{array}{r}83 \\
\pm 5\end{array}$ \\
\hline \multirow[t]{2}{*}{7} & Feb & 8 & $\begin{array}{r}93.8 \\
\pm 4.3\end{array}$ & $\begin{array}{l}45.2 \\
\pm 2.6\end{array}$ & $\begin{array}{r}13.7 \\
\pm 0.6\end{array}$ & $\begin{array}{c}6.6 \\
\pm 0.4\end{array}$ & $\begin{array}{c}0.18 \\
\pm 0.01\end{array}$ & $\begin{array}{c}0.09 \\
\pm 0.01\end{array}$ & $\begin{array}{r}527 \\
\pm 21\end{array}$ & $\begin{array}{r}79 \\
\pm 4\end{array}$ \\
\hline & May & 16 & $\begin{array}{r}65.7 \\
\pm 3.4\end{array}$ & $\begin{array}{r}25.8 \\
\pm 2.8\end{array}$ & $\begin{array}{r}11.7 \\
\pm 0.4\end{array}$ & $\begin{array}{r}4.5 \\
\pm 0.4\end{array}$ & $\begin{array}{c}0.17 \\
\pm 0.01\end{array}$ & $\begin{array}{c}0.06 \\
\pm 0.01\end{array}$ & $\begin{array}{l}399 \\
\pm 9\end{array}$ & $\begin{array}{r}74 \\
\pm 2 \\
\end{array}$ \\
\hline \multirow[t]{4}{*}{8} & Aug & 12 & $\begin{array}{r}32.5 \\
+1.9\end{array}$ & $\begin{array}{r}17.9 \\
\pm 1.7\end{array}$ & $\begin{array}{c}6.4 \\
\pm 0.4\end{array}$ & $\begin{array}{c}3.6 \\
\pm 0.4\end{array}$ & $\begin{array}{c}0.18 \\
\pm 0.01\end{array}$ & $\begin{array}{c}0.10 \\
\pm 0.01\end{array}$ & $\begin{array}{l}181 \\
\pm 7\end{array}$ & $\begin{array}{r}37 \\
\pm 1\end{array}$ \\
\hline & Oct & 8 & $\begin{array}{r}41.7 \\
\pm 1.1\end{array}$ & $\begin{array}{r}23.4 \\
\pm 1.1\end{array}$ & $\begin{array}{c}8.3 \\
\pm 0.2\end{array}$ & $\begin{array}{c}4.7 \\
\pm 0.2\end{array}$ & $\begin{array}{c}0.18 \\
\pm 0\end{array}$ & $\begin{array}{c}0.10 \\
\pm 0\end{array}$ & $\begin{array}{l}227 \\
\pm 4\end{array}$ & $\begin{array}{r}46 \\
\pm 1\end{array}$ \\
\hline & Feb & 8 & $\begin{array}{r}62.2 \\
\pm 3.6\end{array}$ & $\begin{array}{r}34.5 \\
\pm 2.9\end{array}$ & $\begin{array}{r}11.6 \\
\pm 0.5\end{array}$ & $\begin{array}{c}6.4 \\
\pm 0.5\end{array}$ & $\begin{array}{c}0.24 \\
\pm 0.02\end{array}$ & $\begin{array}{c}0.13 \\
\pm 0.01\end{array}$ & $\begin{array}{l}260 \\
\pm 7\end{array}$ & $\begin{array}{l}50 \\
\pm 2\end{array}$ \\
\hline & May & 8 & $\begin{array}{r}47.4 \\
\pm 3.4\end{array}$ & $\begin{array}{r}17.9 \\
\pm 1.7\end{array}$ & $\begin{array}{r}8.5 \\
\pm 0.5\end{array}$ & $\begin{array}{c}3.6 \\
\pm 0.4\end{array}$ & $\begin{array}{c}0.22 \\
\pm 0.01\end{array}$ & $\begin{array}{c}0.10 \\
\pm 0.01\end{array}$ & $\begin{array}{l}212 \\
\pm 7\end{array}$ & $\begin{array}{r}39 \\
\pm 2\end{array}$ \\
\hline
\end{tabular}


Table 2. Latitudinal variation in photosynthesis-irradiance parameters standardised to area (a; $\mu \mathrm{g} \mathrm{O}_{2} \mathrm{~h}^{-1} \mathrm{~cm}^{-2}$ ) and to biomass (b; $\left.\mu \mathrm{g} \mathrm{O}_{2} \mathrm{~h}^{-1} \mathrm{mgC}^{-1}\right), 1988$. Data are means $\pm \mathrm{SE}$

\begin{tabular}{|c|c|c|c|c|c|c|c|c|c|c|}
\hline \multirow[t]{2}{*}{ Zone } & \multirow[t]{2}{*}{ Month } & \multirow[t]{2}{*}{$\mathrm{n}$} & \multicolumn{2}{|c|}{$P_{\max }$} & \multicolumn{2}{|c|}{$R$} & \multicolumn{2}{|c|}{$\alpha\left(=P_{\max } / I_{\mathrm{k}}\right)$} & \multirow{2}{*}{\multicolumn{2}{|c|}{ 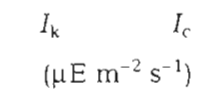 }} \\
\hline & & & $\mathrm{a}$ & $b$ & a & $\mathrm{b}$ & a & b & & \\
\hline \multicolumn{11}{|c|}{ MacGillivray Reef } \\
\hline 7 & Feb & 12 & $\begin{array}{r}70.7 \\
\pm 3.9\end{array}$ & $\begin{array}{r}25.7 \\
\pm 1.7\end{array}$ & $\begin{array}{r}11.8 \\
\pm 0.5\end{array}$ & $\begin{array}{r}4.3 \\
\pm 0.2\end{array}$ & $\begin{array}{r}0.18 \\
\pm 0.01\end{array}$ & $\begin{array}{l}0.06 \\
\pm 0\end{array}$ & $\begin{array}{r}407 \\
\pm 13\end{array}$ & $\begin{array}{r}72 \\
\pm 4\end{array}$ \\
\hline 8 & Feb & 12 & $\begin{array}{r}53.3 \\
\pm 1.2\end{array}$ & $\begin{array}{r}30.5 \\
\pm 1.5\end{array}$ & $\begin{array}{r}9.4 \\
\pm 0.3\end{array}$ & $\begin{array}{r}5.3 \\
\pm 0.2\end{array}$ & $\begin{array}{r}0.24 \\
\pm 0.01\end{array}$ & $\begin{array}{l}0.14 \\
\pm 0\end{array}$ & $\begin{array}{l}220 \\
\pm 6\end{array}$ & $\begin{array}{r}39 \\
\pm 1\end{array}$ \\
\hline \multicolumn{11}{|c|}{ Yonge Reef } \\
\hline \multirow[t]{2}{*}{7} & Feb & 12 & $\begin{array}{r}52.7 \\
\pm 3.0\end{array}$ & $\begin{array}{r}22.8 \\
\pm 1.2\end{array}$ & $\begin{array}{r}9.5 \\
\pm 0.4\end{array}$ & $\begin{array}{r}4.1 \\
\pm 0.2\end{array}$ & $\begin{array}{r}0.16 \\
\pm 0.01\end{array}$ & $\begin{array}{r}0.07 \\
\pm 0.01\end{array}$ & $\begin{array}{r}341 \\
\pm 17\end{array}$ & $\begin{array}{r}65 \\
\pm 4\end{array}$ \\
\hline & Jul & 7 & $\begin{array}{r}46.0 \\
\pm 5.4\end{array}$ & $\begin{array}{r}17.2 \\
\pm 1.0\end{array}$ & $\begin{array}{r}9.4 \\
\pm 1.0\end{array}$ & $\begin{array}{r}3.6 \\
\pm 0.1\end{array}$ & $\begin{array}{r}0.20 \\
\pm 0.02\end{array}$ & $\begin{array}{l}0.07 \\
\pm 0\end{array}$ & $\begin{array}{r}233 \\
\pm 6\end{array}$ & $\begin{array}{r}51 \\
\pm 2\end{array}$ \\
\hline \multirow[t]{2}{*}{8} & Feb & 12 & $\begin{array}{r}46.0 \\
\pm 1.5\end{array}$ & $\begin{array}{r}32.7 \\
\pm 1.0\end{array}$ & $\begin{array}{r}7.8 \\
\pm 0.3\end{array}$ & $\begin{array}{r}5.6 \\
\pm 0.2\end{array}$ & $\begin{array}{r}0.22 \\
\pm 0.01\end{array}$ & $\begin{array}{r}0.15 \\
\pm 0.01\end{array}$ & $\begin{array}{l}215 \\
\pm 9\end{array}$ & $\begin{array}{r}37 \\
\pm 1\end{array}$ \\
\hline & Jul & 11 & $\begin{array}{r}41.0 \\
\pm 2.1\end{array}$ & $\begin{array}{r}27.8 \\
\pm 1.7\end{array}$ & $\begin{array}{r}7.0 \\
\pm 0.3\end{array}$ & $\begin{array}{r}4.8 \\
\pm 0.3\end{array}$ & $\begin{array}{r}0.21 \\
\pm 0.01\end{array}$ & $\begin{array}{r}0.15 \\
\pm 0.01\end{array}$ & $\begin{array}{r}208 \\
\pm 24\end{array}$ & $\begin{array}{r}45 \\
\pm 5\end{array}$ \\
\hline \multicolumn{11}{|c|}{ Davies Reef } \\
\hline \multirow[t]{2}{*}{7} & Feb & 12 & $\begin{array}{r}51.5 \\
\pm 2.8\end{array}$ & $\begin{array}{r}22.3 \\
\pm 2.1\end{array}$ & $\begin{array}{r}8.7 \\
\pm 0.5\end{array}$ & $\begin{array}{r}3.8 \\
\pm 0.3\end{array}$ & $\begin{array}{r}0.14 \\
\pm 0.01\end{array}$ & $\begin{array}{r}0.06 \\
\pm 0.01\end{array}$ & $\begin{array}{r}386 \\
\pm 18\end{array}$ & $\begin{array}{r}67 \\
\pm 5\end{array}$ \\
\hline & Jul & 7 & $\begin{array}{r}41.3 \\
\pm 2.4\end{array}$ & $\begin{array}{r}14.2 \\
\pm 1.9\end{array}$ & $\begin{array}{r}7.2 \\
\pm 0.3\end{array}$ & $\begin{array}{r}2.4 \\
\pm 0.3\end{array}$ & $\begin{array}{r}0.14 \\
\pm 0.01\end{array}$ & $\begin{array}{r}0.05 \\
\pm 0.01\end{array}$ & $\begin{array}{r}309 \\
\pm 15\end{array}$ & $\begin{array}{r}55 \\
\pm 2\end{array}$ \\
\hline \multirow[t]{2}{*}{8} & Feb & 8 & $\begin{array}{r}47.3 \\
\pm 1.4\end{array}$ & $\begin{array}{r}31.8 \\
\pm 8.9\end{array}$ & $\begin{array}{r}8.5 \\
\pm 0.3\end{array}$ & $\begin{array}{r}5.7 \\
\pm 0.3\end{array}$ & $\begin{array}{r}0.25 \\
\pm 0.01\end{array}$ & $\begin{array}{r}0.17 \\
+0.01\end{array}$ & $\begin{array}{l}189 \\
\pm 7\end{array}$ & $\begin{array}{r}34 \\
\pm 1\end{array}$ \\
\hline & Jul & 11 & $\begin{array}{r}33.0 \\
\pm 1.1\end{array}$ & $\begin{array}{r}17.9 \\
\pm 1.4\end{array}$ & $\begin{array}{r}5.5 \\
\pm 0.3\end{array}$ & $\begin{array}{r}3.0 \\
\pm 0.2\end{array}$ & $\begin{array}{r}0.21 \\
\pm 0.02\end{array}$ & $\begin{array}{r}0.11 \\
\pm 0.01\end{array}$ & $\begin{array}{r}170 \\
\pm 13\end{array}$ & $\begin{array}{r}28 \\
\pm 1\end{array}$ \\
\hline \multicolumn{11}{|c|}{ Myrmidon Reef } \\
\hline \multirow[t]{2}{*}{7} & Feb & 12 & $\begin{array}{r}52.2 \\
\pm 3.0\end{array}$ & $\begin{array}{r}17.8 \\
\pm 0.8\end{array}$ & $\begin{array}{r}10.7 \\
\pm 0.5\end{array}$ & $\begin{array}{r}3.7 \\
\pm 0.2\end{array}$ & $\begin{array}{r}0.14 \\
\pm 0.01\end{array}$ & $\begin{array}{l}0.05 \\
\pm 0\end{array}$ & $\begin{array}{r}379 \\
\pm 23\end{array}$ & $\begin{array}{r}81 \\
\pm 4\end{array}$ \\
\hline & Jul & 8 & $\begin{array}{r}42.6 \\
\pm 6.9\end{array}$ & $\begin{array}{r}11.6 \\
\pm 1.7\end{array}$ & $\begin{array}{r}8.7 \\
\pm 0.9\end{array}$ & $\begin{array}{r}2.4 \\
\pm 0.2\end{array}$ & $\begin{array}{r}0.10 \\
\pm 0.02\end{array}$ & $\begin{array}{l}0.03 \\
\pm 0\end{array}$ & $\begin{array}{r}458 \\
\pm 30\end{array}$ & $\begin{array}{r}103 \\
\pm 10\end{array}$ \\
\hline \multirow[t]{2}{*}{8} & Feb & 12 & $\begin{array}{r}45.5 \\
\pm 1.8\end{array}$ & $\begin{array}{r}25.7 \\
\pm 0.8\end{array}$ & $\begin{array}{r}8.8 \\
\pm 0.5\end{array}$ & $\begin{array}{r}4.9 \\
\pm 0.2\end{array}$ & $\begin{array}{r}0.19 \\
\pm 0.01\end{array}$ & $\begin{array}{r}0.11 \\
\pm 0.01\end{array}$ & $\begin{array}{r}237 \\
\pm 8\end{array}$ & $\begin{array}{r}47 \\
\pm 1\end{array}$ \\
\hline & Jul & 8 & $\begin{array}{r}31.4 \\
+4.5\end{array}$ & $\begin{array}{r}21.0 \\
\pm 2.5\end{array}$ & $\begin{array}{r}5.5 \\
+0.9\end{array}$ & $\begin{array}{r}3.6 \\
\pm 0.6\end{array}$ & $\begin{array}{r}0.19 \\
\pm 0.02\end{array}$ & $\begin{array}{r}0.13 \\
\pm 0.01\end{array}$ & $\begin{array}{r}165 \\
\pm 14\end{array}$ & $\begin{array}{r}28 \\
\pm 2\end{array}$ \\
\hline \multicolumn{11}{|c|}{ Heron Island } \\
\hline \multirow[t]{2}{*}{7} & Feb & 12 & $\begin{array}{r}61.2 \\
+2.3\end{array}$ & $\begin{array}{r}24.5 \\
+1.8\end{array}$ & $\begin{array}{r}10.6 \\
\pm 0.2\end{array}$ & $\begin{array}{r}4.2 \\
\pm 0.2\end{array}$ & $\begin{array}{l}0.12 \\
\pm 0\end{array}$ & $\begin{array}{l}0.05 \\
\pm 0\end{array}$ & $\begin{array}{r}498 \\
\pm 18\end{array}$ & $\begin{array}{r}91 \\
\pm 3\end{array}$ \\
\hline & Jul & 7 & $\begin{array}{r}37.7 \\
\pm 1.8\end{array}$ & $\begin{array}{r}10.3 \\
+1.2\end{array}$ & $\begin{array}{r}7.8 \\
\pm 0.2\end{array}$ & $\begin{array}{r}2.1 \\
\pm 0.2\end{array}$ & $\begin{array}{r}0.19 \\
\pm 0.02\end{array}$ & $\begin{array}{r}0.05 \\
\pm 0.01\end{array}$ & $\begin{array}{r}208 \\
\pm 24\end{array}$ & $\begin{array}{r}45 \\
\pm 5\end{array}$ \\
\hline \multirow[t]{2}{*}{8} & Feb & 12 & $\begin{array}{r}49.0 \\
\pm 1.9\end{array}$ & $\begin{array}{r}34.7 \\
\pm 1.2\end{array}$ & $\begin{array}{r}8.4 \\
+0.5\end{array}$ & $\begin{array}{r}6.0 \\
\pm 0.5\end{array}$ & $\begin{array}{r}0.22 \\
\pm 0.01\end{array}$ & $\begin{array}{r}0.16 \\
\pm 0.01\end{array}$ & $\begin{array}{l}219 \\
\pm 6\end{array}$ & $\begin{array}{r}38 \\
\pm 2\end{array}$ \\
\hline & Jul & 4 & $\begin{array}{r}29.0 \\
\pm 3.7\end{array}$ & $\begin{array}{r}19.6 \\
\pm 0.7\end{array}$ & $\begin{array}{r}4.4 \\
\pm 0.6\end{array}$ & $\begin{array}{r}3.0 \\
\pm 0.2\end{array}$ & $\begin{array}{r}0.22 \\
\pm 0.03\end{array}$ & $\begin{array}{r}0.15 \\
\pm 0.01\end{array}$ & $\begin{array}{r}137 \\
\pm 16\end{array}$ & $\begin{array}{r}21 \\
\pm 2\end{array}$ \\
\hline \multicolumn{11}{|c|}{ One Tree Island } \\
\hline 7 & Feb & 10 & $\begin{array}{r}50.5 \\
\pm 2.4\end{array}$ & $\begin{array}{r}20.3 \\
\pm 2.6\end{array}$ & $\begin{array}{r}9.9 \\
\pm 0.3\end{array}$ & $\begin{array}{r}3.9 \\
\pm 0.4\end{array}$ & $\begin{array}{r}0.17 \\
\pm 0.01\end{array}$ & $\begin{array}{r}0.07 \\
\pm 0.01\end{array}$ & $\begin{array}{r}315 \\
\pm 26\end{array}$ & $\begin{array}{r}64 \\
\pm 4\end{array}$ \\
\hline 7 & Jul & 12 & $\begin{array}{r}34.9 \\
\pm 1.8\end{array}$ & $\begin{array}{r}8.4 \\
\pm 0.7\end{array}$ & $\begin{array}{r}6.9 \\
\pm 0.2\end{array}$ & $\begin{array}{r}1.7 \\
\pm 0.1\end{array}$ & $\begin{array}{r}0.17 \\
\pm 0.01\end{array}$ & $\begin{array}{l}0.04 \\
\pm 0\end{array}$ & $\begin{array}{r}209 \\
\pm 8\end{array}$ & $\begin{array}{r}42 \\
\pm 1\end{array}$ \\
\hline \multirow[t]{2}{*}{8} & $\mathrm{Feb}$ & 10 & $\begin{array}{r}51.4 \\
+1.8\end{array}$ & $\begin{array}{r}28.3 \\
+2.6\end{array}$ & $\begin{array}{r}9.3 \\
\pm 0.5\end{array}$ & $\begin{array}{r}5.1 \\
\pm 0.5\end{array}$ & $\begin{array}{r}0.24 \\
\pm 0.01\end{array}$ & $\begin{array}{r}0.14 \\
\pm 0.02\end{array}$ & $\begin{array}{r}211 \\
\pm 8\end{array}$ & $\begin{array}{r}39 \\
\pm 2\end{array}$ \\
\hline & Jul & 12 & $\begin{array}{r}36.1 \\
\pm 1.9\end{array}$ & $\begin{array}{r}16.9 \\
\pm 1.6\end{array}$ & $\begin{array}{r}7.1 \\
\pm 0.6\end{array}$ & $\begin{array}{r}3.2 \\
\pm 0.3\end{array}$ & $\begin{array}{r}0.25 \\
\pm 0.01\end{array}$ & $\begin{array}{r}0.12 \\
\pm 0.01\end{array}$ & $\begin{array}{r}154 \\
\pm 14\end{array}$ & $\begin{array}{r}33 \\
\pm 5\end{array}$ \\
\hline
\end{tabular}


The parameter $\alpha$ a measure of the efficiency with which light is utilised by the photosynthetic system, changed little between either seasons or reefs, and only varied significantly with depth; consistently higher values were found for the EAC of reef slopes compared with those of reef flat communities, and this was most evident in biomass-specific terms (Tables 1 \& 2 ). These results are consistent with the observation that shade algae are characterised by relatively high alpha values. Generally, there were no interannual differences in P-I parameters of the EAC (Tables $1 \& 2$ ) from comparable reefs (Davies or Myrmidon), habitats (Zone 7 or 8 ) and seasons (winter or summer).

\section{Productivity of EAC on coral plates}

Production of the EAC based both on area $\left(P_{\mathrm{a}}\right)$ and biomass $\left(P_{\mathrm{b}}\right)$ varied strongly with season (F-test, $\mathrm{p}<$ 0.0001 ) for all zones and reefs of the cross-shelf (Fig. 6) and latitudinal transects (Fig. 7). Productivity was highest in summer and lowest in winter (SNK test). Considering the cross-shelf transect, reef location generally had no significant effect ( $F$-test) on $P_{\mathrm{a}}$ and $P_{\mathrm{b}}$ of EAC on either reef flats or reef slopes (Fig. 6). There was a higher rate of productivity on Myrmidon Reef flat than on Davies and Pandora Reefs in summer 1987 a
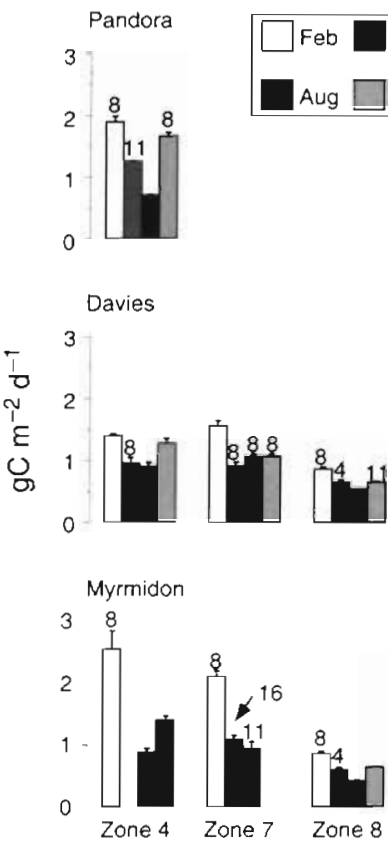

b
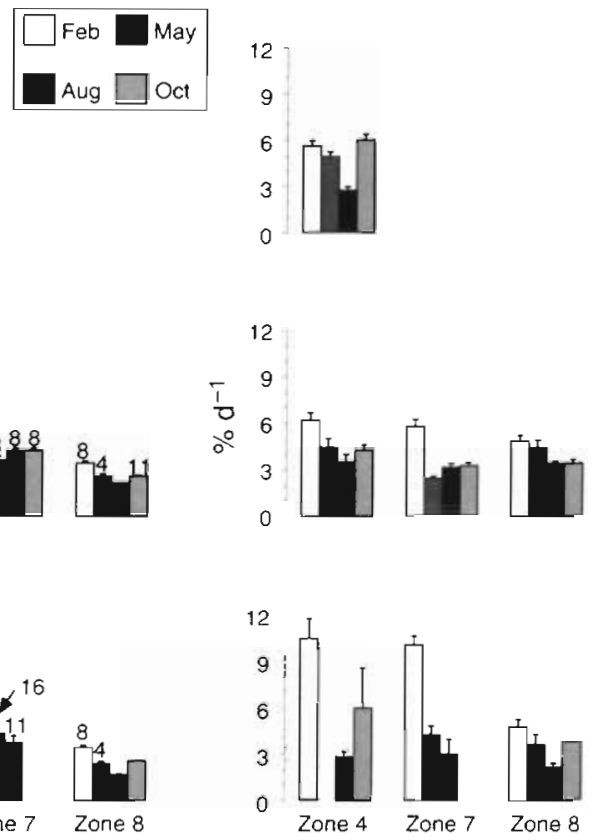

Fig. 6. Spatial and temporal variation in net primary production standardised (a) to area and (b) to biomass on reefs of the cross-shelf transect. Data are means $+\mathrm{SE}_{\mathrm{i}} \mathrm{n}=12$, or as given above bars

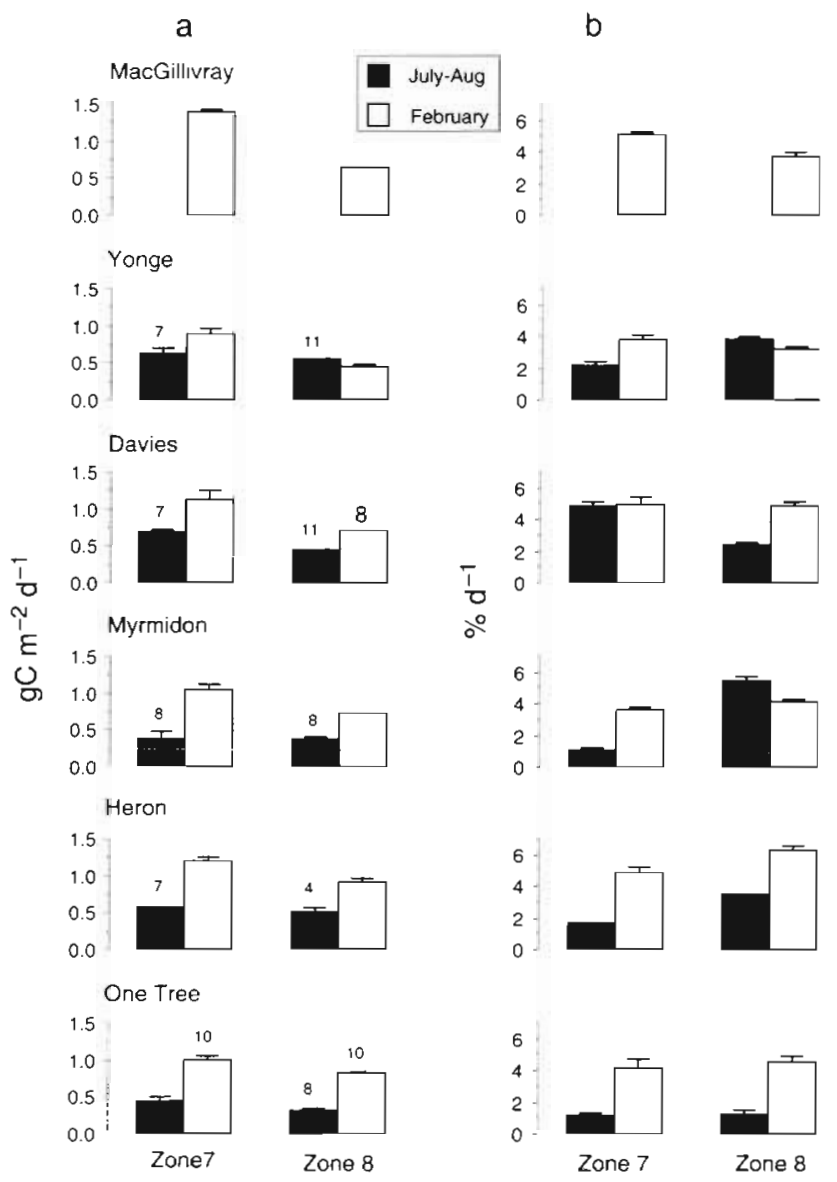

Fig. 7. Spatial and temporal variation in the net primary production standardised (a) to area and (b) to biomass on reefs of the latitudinal transect. Data are means $+\mathrm{SE}_{i} \mathrm{n}=12$, or as given above bars

(reef slopes were not different), but this was not evident in the following summer (latitudinal transect), when productivity on Myrmidon Reef had decreased to more typical levels $\left(P_{\mathrm{a}}=1.0\right.$ to $1.5 \mathrm{~g} \mathrm{C} \mathrm{m}^{-2} \mathrm{~d}^{-1}$; Fig. 7). Moreover, in 1988, the latitudinal transect (which also included a comparison of mid-and outershelf reef location) showed no significant intra-reef patterns in EAC production ( $F$-test). In all seasons, $P_{\mathrm{b}}$ was the same on reef flats and slopes ( $F$-test), but because of the differences in biomass, EAC of reef flat habitats had significantly higher $\mathrm{P}_{\mathrm{a}}(F$-test, $\mathrm{p}<0.0001)$ than the community on the adjacent reef slopes.

Mean $P_{\mathrm{a}}$ of EAC on coral plates, averaged over all reefs, winter and summer, 1987 and 1988, was $1.1 \mathrm{~g} \mathrm{C}$ $\mathrm{m}^{-2} \mathrm{~d}^{-1}$ (winter: 0.7, summer: $1.5 \mathrm{~g} \mathrm{C} \mathrm{m}^{-2} \mathrm{~d}^{-1}$ ) on reef flats and $0.6 \mathrm{~g} \mathrm{C} \mathrm{m}^{-2} \mathrm{~d}^{-1}$ (winter: 0.4 , summer: $0.8 \mathrm{~g} \mathrm{C}$ $\mathrm{m}^{-2} \mathrm{~d}^{-1}$ ) on reef slopes. Thus, annual production of the EAC (without taking EAC coverage, or irregularity of the reef surface into account) is $400 \mathrm{~g} \mathrm{C} \mathrm{m}^{-2}$ on reef flats and $220 \mathrm{~g} \mathrm{C} \mathrm{m}^{-2}$ on reef slopes. 


\section{Effect of community structure and biomass on EAC productivity}

The percent cover of the plates by crustose coralline algae $(C A)$, had a strong effect on the biomass $(B, \mathrm{~g} C$ $\mathrm{m}^{-2}$; see Fig. 4) of the EAC on reef flats of the crossshelf and latitudinal transects. The regressions of $B$ on $C A$ were expressed by $B=18.2+0.2 C A$ ( $\mathrm{r}=0.54$, p < $0.001, \mathrm{n}=40)$ in summer, and $B=24.9+0.2 C A(\mathrm{r}=$ $0.69, \mathrm{p}<0.001, \mathrm{n}=59$ ) in winter (cross-shelf and latitudinal data combined). Thus, the fine turf component of the EAC on the reef flat had a predicted biomass of 18.2 and $24.9 \mathrm{~g} \mathrm{C} \mathrm{m}^{-2}$ in summer and winter, respectively, i.e. ca $50 \%$ of the biomass of the crustose coralline algal component. On reef slopes, there was no significant correlation $(\mathrm{r}=0.06, \mathrm{n}=65)$ between $B$ and $C A$, in any season.

There was also a highly significant inverse relationship between coralline algal cover and biomassspecific production $\left(P_{\mathrm{b}}\right)$ of the EAC on reef flats in both summer $(\mathrm{r}=0.60, \mathrm{p}<0.001, \mathrm{n}=40)$ and winter $(r=0.46, p<0.005, n=39)$. The slopes of these relationships did not differ significantly between seasons (ANCOVA), though $P_{\mathrm{b}}$ of both coralline and turf algae decreased from summer to winter. Predicted $P_{\mathrm{b}}$ of pure coralline algae would be 1.3 and $2.4 \% \mathrm{~d}^{-1}$ in winter and summer, respectively, compared with 3.5 and $6.4 \% \mathrm{~d}^{-1}$ for fine turfs (cross-shelf \& latitudinal data combined). The increase in biomass, and the decrease in $P_{\mathrm{b}}$ of the EAC with an increase in $C A$, as described above, was such that areal production of the total community $\left(P_{\mathrm{a}}\right)$ on reef flats, in both summer and winter, appeared to be independent of the EAC composition. On reef slopes, $C A$ had no significant effect on EAC $P_{\mathrm{b}}$ or biomass, and thus, had no effect on $P_{\mathrm{a}}$.

Algal biomass had a strong negative effect upon biomass-specific production for substrata dominated by fine turf (i.e. samples with $>75 \%$ fine-turf cover), regardless of habitat and season (reef flats, summer: $r=0.43, p<0.05, n=22$; winter: $r=0.68, p<0.002$, $\mathrm{n}=18$; reef slopes, summer: $\mathrm{r}=0.80, \mathrm{p}<0.002, \mathrm{n}=12$; winter: $r=0.62, p<0.005, n=22$ ).

\section{EAC cover and irregularity of reef surfaces}

The EAC (turfs plus coralline algae) covered a high proportion of the surface on all mid-and outer-shelf reefs examined (Table 3 ). There were no significant differences in the total EAC cover of reef slopes ( $F$ test; mean cover $41 \%$, Table 3 ), but the difference between 3 groups of reef flats was highly significant $(F-$ test, $\mathrm{p}<0.0001_{\text {i }}$ SNK test). These groups were Yonge, Davies and Myrmidon Reefs with the highest cover (mean EAC cover $=76 \%$ ), followed by McGillivray, One Tree and Heron Island Reefs (mean $=54 \%$ ), and Pandora Reef with lowest cover (24\%). In general, hard corals were the other major occupant of space on these reefs ( 11 to $44 \%$ on flats; 14 to $35 \%$ on slopes Fig. 8). Although there were some significant differences between reefs in terms of the cover of particular types of EAC (F-test, SNK test; e.g. coralline algal cover on Yonge Reef higher than other reefs), these did

Table 3. Coverage by EAC (turfs plus coralline algae; in percent) and irregularity of reef surfaces. Data are means $\pm \mathrm{SE}$

\begin{tabular}{|c|c|c|c|c|}
\hline \multirow[t]{2}{*}{ Reef } & \multicolumn{2}{|c|}{ EAC cover } & \multicolumn{2}{|c|}{ Irregularity factor ${ }^{\mathrm{d}}$} \\
\hline & $\begin{array}{l}\text { Reef } \\
\text { flat }\end{array}$ & $\begin{array}{l}\text { Reef } \\
\text { slope }\end{array}$ & $\begin{array}{c}\text { Reef } \\
\text { flat }\end{array}$ & $\begin{array}{l}\text { Reef } \\
\text { slope }\end{array}$ \\
\hline MacGilliviay & $\begin{array}{r}57 \\
\pm 2\end{array}$ & $\begin{array}{r}38 \\
\pm 4\end{array}$ & $\begin{array}{r}1.25 \\
\pm 0.02\end{array}$ & $\begin{array}{r}1.16 \\
\pm 0.04\end{array}$ \\
\hline Yonge & $\begin{array}{r}81 \\
\pm 3\end{array}$ & $\begin{array}{r}53 \\
\pm 5\end{array}$ & $\begin{array}{r}1.30 \\
\pm 0.02\end{array}$ & $\begin{array}{r}1.52 \\
\pm 0.06\end{array}$ \\
\hline Pandora & $\begin{array}{r}24 \\
\pm 4\end{array}$ & - & $\begin{array}{r}1.27 \\
\pm 0.02\end{array}$ & - \\
\hline Davies & $\begin{array}{r}73 \\
\pm 5\end{array}$ & $\begin{array}{r}44 \\
\pm 5\end{array}$ & $\begin{array}{r}1.30 \\
\pm 0.03\end{array}$ & $\begin{array}{r}1.33 \\
\pm 0.04\end{array}$ \\
\hline Myrmidon & $\begin{array}{r}74 \\
\pm 3\end{array}$ & $\begin{array}{r}38 \\
\pm 3\end{array}$ & $\begin{array}{r}1.16 \\
\pm 0.02\end{array}$ & $\begin{array}{r}1.33 \\
\pm 0.03\end{array}$ \\
\hline Heron & $\begin{array}{r}51 \\
\pm 3\end{array}$ & $\begin{array}{r}35 \\
\pm 1\end{array}$ & $\begin{array}{r}1.30 \\
\pm 0.01\end{array}$ & $\begin{array}{r}1.19 \\
\pm 0.03\end{array}$ \\
\hline One Tree & $\begin{array}{r}55 \\
\pm 3\end{array}$ & $\begin{array}{r}33 \\
\pm 8\end{array}$ & $\begin{array}{r}1.29 \\
\pm 0.02\end{array}$ & $\begin{array}{r}1.13 \\
\pm 0.03\end{array}$ \\
\hline $\begin{array}{l}\text { 'Briefly this } \\
\text { across the r } \\
\text { length over } \\
\text { methods' for }\end{array}$ & $\begin{array}{l}\text { obtair } \\
\text { surfac } \\
\text { ight } 1 \\
\text { ails }\end{array}$ & $\begin{array}{l}d \text { by } r \\
\text { then } t \\
\text { e dista }\end{array}$ & $\begin{array}{l}\text { a lengt } \\
\text { the rati } \\
\text { see 'Mat }\end{array}$ & $\begin{array}{l}\text { of chain } \\
\text { of chain } \\
\text { lals and }\end{array}$ \\
\hline
\end{tabular}

not suggest any latitudinal trends, nor any consistent differences between mid- and outer-shelf reefs. The one inner-shelf reef examined in this study (Pandora Reef) was distinctive in being dominated by zooanthids (61\%; Fig. 8), as well as its comparatively low EAC coverage. Moreover, damselfish-territory turf dominated the EAC (75\%) on the natural surfaces of Pandora Reef, as was observed with coral plates from this reef, whereas in comparable habitats on mid-and outer-shelf reefs, fine turfs and coralline algae were equally important components of the EAC. Reef flats had consistently higher cover of EAC (range: 51 to $81 \%$; Table 3 ) than the adjacent reef slopes (range: 33 to $73 \%$; Table 3 ), which had extensive areas of sand (Fig. 8). In addition, coralline algae were important on reef flats, and filamentous algae dominated the reef slopes (Fig. 8). 


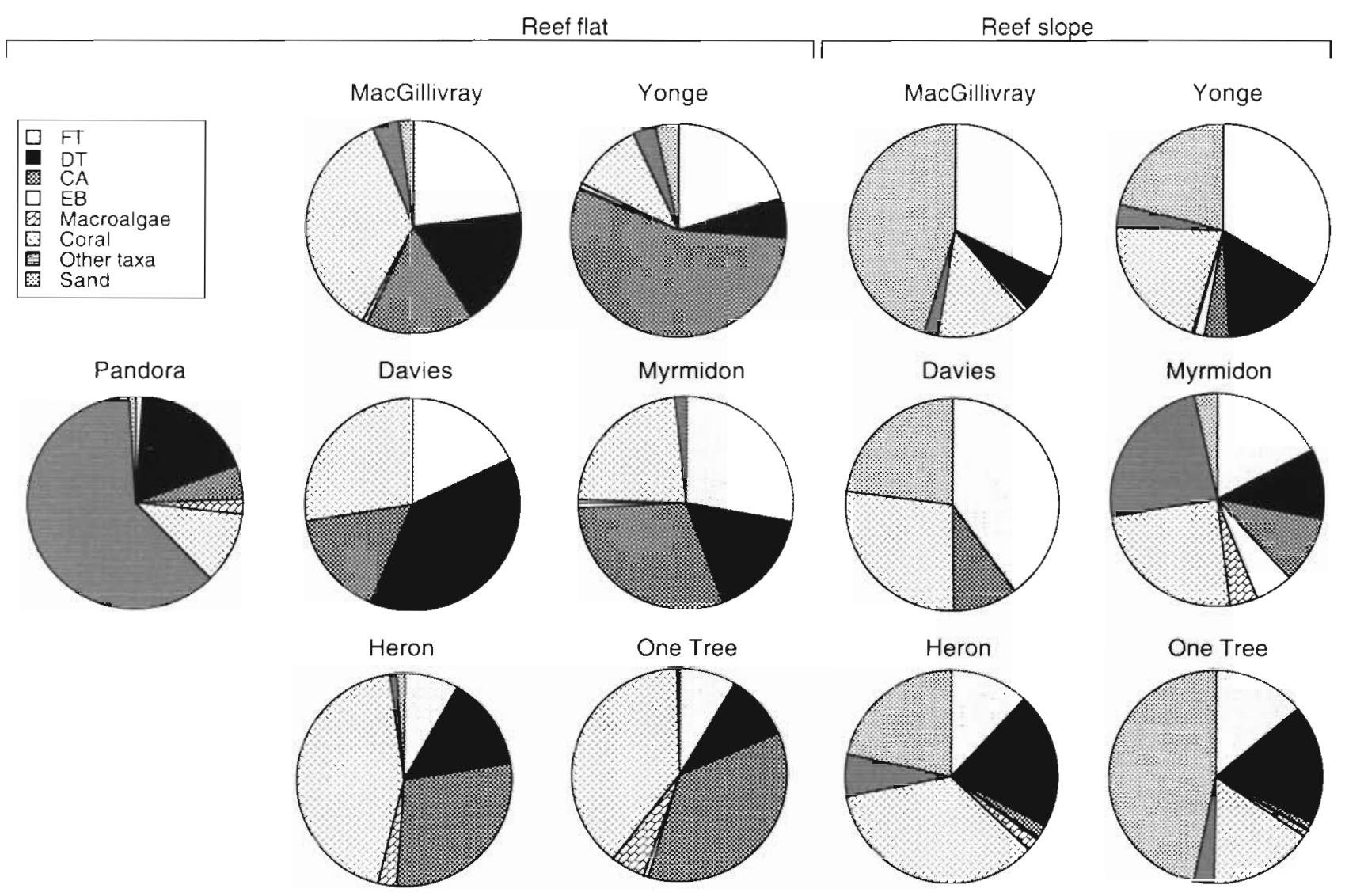

Fig. 8. Composition of the reef substratum (expressed as mean percent cover) of the reef flats and reef slopes on the cross-shelf and latitudinal transects. Algal type codes as in Fig. 2

There were no significant intra-reef differences in the irregularity factor $(I F)$ of the surface on reef slopes ( $F$-test, $\mathrm{p}=0.07$; mean $I F=1.28$, Table 3 ). On reef flats, only Myrmidon Reef had a significantly lower IF ( $F$ test, $\mathrm{p}=0.03$; SNK test $i F=1.16$ ) than the other reefs (mean $I F=1.28$; Table 3 ). Thus, generally reef flats and reef slopes had similar $I F^{\prime}$ s.

\section{Productivity of EAC per unit area of reef surface}

The expected annual net production of EAC per unit area of reef surface on reef flats and slopes for selected reefs of the GBR was calculated as the product of mean annual $P_{\mathrm{a}}$ for the EAC on experimental coral plates, the proportional coverage of the natural reef surface by $E A C$, and the irregularity of the reef surface (i.e. $I F^{2}$ ). Rates of production (in $\mathrm{g} \mathrm{C} \mathrm{m}^{-2} \mathrm{yr}^{-1}$ ) were 148 on all reef slopes, and on reef flats were 157 for Pandora Reef, 354 for McGillivray, One Tree Island and Heron Island Reefs, 410 for Myrmidon Reef, and 500 for Yonge and Davies Reefs.

\section{DISCUSSION}

This study provides the first quantification of the variation in the structure, biomass and productivity of the epilithic algal community (EAC) on a number of reefs over a wide area of the Great Barrier Reef (GBR). In addition, it describes within-reef and seasonal variability of these parameters. The temporal and spatial patterns in the community structure, biomass and metabolism which characterised the EAC of 1 reef on the mid-shelf, central section of the Great Barrier Reef (Davies Reef; Klumpp \& McKinnon 1989) are found to be generally applicable to other reefs in this region.

The EAC covers a high proportion of reef flats (up to $80 \%$ ) and reef slopes (up to $50 \%$ ) throughout the GBR. However, the extent of this cover, as well as the community structure of the EAC, varies with location, such that cover of substrata by algae is greatest on reef flats in the mid-and outer-shelf region, where the community is usually dominated by a mixture of wellgrazed turfs and crustose coralline algae. Crustose coralline algae, considered to be the algal group most 
adapted to intense grazing (Steneck 1985, Hackney et al. 1989), are relatively abundant on reef flats, especially around the crests of reefs $(69 \pm 15 \%$ cover of plates averaged over all reefs; see also Klumpp \& Mckinnon 1989), where grazing activity is most intense (Hatcher 1982, Klumpp \& Polunin 1990). Cover of reef surfaces by EAC declines, and a shift to a simpler, turf-dominated community occurs with increasing depth (see also Klumpp \& McKinnon 1989), and the nearness of reefs to the coast. Scott \& Russ (1987) reported a similar cross-shelf pattern of EAC structure on the leeward crests of reefs in the central section of the GBR.

Large fleshy algae (e.g. Sargassum spp.), which are usually relatively rare in all habitats on the mid-and outer-shelf reefs (Borowitzka \& Larkum 1986, Bradbury et al. 1986), dominate in some seasons and habitats on inner-shelf reefs (Morrissey 1980, Done 1982, Klumpp unpubl.). Although large fleshy algae were not common in the study site at Pandora Reef, the adjacent windward edge of this reef has an extensive Sargassum community. The transition from an algal community, comprising mainly coralline and low turfs, on reefs of the mid-and outer-shelf to one characterised by lush turfs and large fleshy algae correlates with a significant decrease in grazing intensity (see Williams et al. 1986).

The clear pattern of changes in algal community structure observed across the continental shelf and down a depth-profile, were not evident on the larger scale of the latitudinal transect. Studies on the abundance and composition of other reef communities (corals: Done 1982; Halimeda spp.: Drew (1983); sponges: Wilkinson 1986; herbivorous fishes: Williams et al. 1986) also report strong patterns of cross-shelf variation, and, at least for the fishes, this greatly exceeds the variation on the latitudinal gradient.

Variations in biomass (except in winter), and net primary production of the EAC per se on the GBR show no apparent cross-shelf, or latitudinal patterns, and a comparison of habitats at the same depth within one reef (e.g. Zone 4 cf. Zone 7) shows no significant differences in these parameters (see also Klumpp \& Mckinnon 1989). Moreover, interannual variation (comparison of 1987 and 1988 on Davies and Myrmidon Reefs) in productivity and P-I parameters was generally not significant. Rather, these parameters vary more with depth and season in a fairly predictable manner; maximum areal productivity occurs in summer in shallow habitats and minimum productivity occurs in winter on the reef slope. To some extent, these variations in EAC productivity can be attributed to changes in irradiance, but the response of photosynthetic rate to irradiance is complicated due to photoadaptation by the EAC under conditions of reduced irradiance, as evident in the seasonal and depthrelated changes in the P-I parameters, $I_{\mathrm{k}}, P_{\text {max }}$, and $\alpha_{\text {, }}$ of the EAC. For example, productivity per weight of epilithic algae in all habitats examined decreases significantly from summer to winter with the decrease in irradiance, but over this period the $I_{k}$ and $I_{c}$ values also decrease, showing that the algae are able to alter their photosynthetic apparatus to make more efficient use of available light. In addition, the communities on reef slopes at $10 \mathrm{~m}$, despite the lower irradiance reaching this habitat, are as productive as those in shallow parts of the reef because they are more photosynthetically efficient (high $\alpha$ ).

Patches of crustose coralline algae have a higher biomass, but a lower photosynthetic rate per unit biomass $\left(P_{\mathrm{b}}\right)$ than the equivalent area of fine turf algae (see also Littler \& Arnold 1982, Klumpp \& McKinnon 1989). The net result is that these two algal forms have comparable rates of areal productivity (see also Wanders 1976). Thus, variation in the relative proportions of coralline and turf algae comprising the $\mathrm{EAC}$ is predicted to have an effect upon the biomass and the biomass-specific productivity of the EAC. However, this is complicated by the relative abundance of damselfish territory turf, the third major algal group of the EAC, which is the most productive form of epilithic algae on reefs (see Klumpp et al. 1987). Indeed, a comparison of the midreef flat with the reef crest habitat of Davies and Myrmidon Reefs, between which there are considerable differences in the relative proportions of crustose coralline algae and fine turf algae, show a predicted, though not always significant, difference in biomass and biomass-specific productivity. The biomass of turfs is shown to be another important determinant of productivity of the EAC, since where fine turf dominates the substratum, its productivity is inversely correlated with biomass. Carpenter (1985) also noted this effect in his examination of epilithic algae on a Caribbean reef, and suggested that it is probably related to selfshading.

In shallow reef areas, EAC biomass is lowest in summer and peaks around winter, whereas at $10 \mathrm{~m}$ on the reef slope, biomass is lower than on the reef flat, and it is seasonally stable. The seasonal variation in biomass of EAC on the reef flat can be explained by the seasonal changes in grazing intensity relative to productivity. While variations in productivity and grazing intensity are positively correlated, the magnitude of seasonal change in rate of grazing on algae exceeds that produced (Klumpp \& Polunin 1990), thus resulting in the observed seasonal fluctuations in biomass. On the reef slope, presumably EAC productivity and losses, such as those due to grazing, are in balance over the year. Similarly, Borowitzka (1981) and Hatcher \& Larkum (1983) observed that epilithic algal 
biomass decreased towards summer on the reef flat at One Tree Island, and that this corresponded with an increase in grazing activity. The lower biomass of the $\mathrm{EAC}$ on the reef slope compared with the reef flat is in part due to the difference in community structure; reef slopes are dominated by turfs (ca $80 \%$ cover), which have a lower biomass per unit area than coralline algae.

It is now well established that the EAC represents an extremely important trophic resource on coral reefs through the interaction with grazers (Hatcher 1983, Carpenter 1986, Klumpp \& Polunin 1989, 1990). In turn, grazers exercise a strong influence on the biomass and community structure of reef algal communities (see Hatcher 1983). Grazing activity, within certain limits, is also thought to stimulate productivity of epilithic algae by selecting for fast growing forms of algae, the removal of senescent material and an enhanced availability of nutrients. Over an entire reef flat, it has been estimated that grazers account for 40 to $70 \%$ of EAC net production, but there is considerable spatial variability in grazing intensity (Klumpp \& Polunin 1990). For example, grazing rates are highest on the outer shallow reef crests and slopes, and lowest in leeward edges of reef flats and in lagoons. On a larger scale, the abundance of herbivorous fishes (the major grazers on the GBR; Klumpp \& Polunin 1989, 1990), and the rate of removal of the EAC by grazers (determined by grazer exclusion experiments) is much higher on mid- and outer-shelf reefs than on reefs nearshore (see Williams et al. 1986). These differences in grazing pressure within reefs, and between reefs located across the GBR, do not influence the rate of turnover of the epilithic algae (i.e. that established on coral plates) being grazed. For example, algal communities from different habitats at the same depth on Davies Reef are equally productive (Klumpp \& McKinnon 1989). Furthermore, the EAC on Pandora Reef was as productive per unit area and per unit biomass as the communities on Myrmidon and Davies Reefs.

The rate of EAC production per unit area of reef surface, or actual algal food availability (i.e. the areal production of EAC on coral plates corrected for the irregularity and algal coverage of reef surfaces) does, however, correlate with the rate at which algae are removed by grazers, as was demonstrated in the comparison between reef-flat habitats on Davies Reef (Klumpp \& McKinnon 1989, Klumpp \& Polunin 1990). Similarly, in considering the cross-shelf gradient, the availability of algal food resources on Davies and Myrmidon Reefs ( 400 to $500 \mathrm{~g} \mathrm{C} \mathrm{m}^{-2} \mathrm{yr}^{-1}$ ) is 3 times that of Pandora Reef ( $\left.160 \mathrm{~g} \mathrm{C} \mathrm{m}^{-2} \mathrm{yr}^{-1}\right)$. This compares with a 3- to 5-fold differential in abundance of grazers on these 2 groups of reefs (Williams et al. 1986). These differences in algal food availability between sites are due to differences in algal coverage of reef surfaces. Williams and co-workers estimated grazing rate at ca $0.1 \mathrm{~g} \mathrm{C} \mathrm{m}^{-2} \mathrm{~d}^{-1}$ on Pandora Reef and ca $0.6 \mathrm{~g} \mathrm{C} \mathrm{m}^{-2}$ $\mathrm{d}^{-1}$ on the mid- and outer-shelf reefs. Hence, grazing accounts for $23 \%$ of the EAC net production on Pandora Reef and approximately $50 \%$ of the production on the reefs offshore. This is in close agreement with independent estimates of the average annual grazing impact on the EAC at Davies Reef $157 \%$; Klumpp \& Polunin 1990) and on One Tree Island (50\%; Hatcher 1982). It remains unknown, as pointed out by Williams et al. (1986), whether grazer abundance controls algal food availability, or vice versa.

Acknowledgements. We thank the staff of the Engineering and Electronics Workshops at AIMS for their contributions to the development and construction of the respirometers used in this study. John Collingwood's efforts were particularly appreciated. We are also grateful to the crews of RV 'The Harry Messel' and RV 'Lady Basten', and the many volunteer divers for their assistance with the field sampling programme.

\section{LITERATURE CITED}

Borowitzka, M. A. (1981). A.lgae and grazing in coral reef ecosystems. Endeavour 5: 99-106

Borowitzka, M. A., Day, R., Larkum, A. W. D. (1983). The importance of primary production by turf and crustose algal communities in One Tree Lagoon. In: Baker, J. T., Carter, R. M., Sammarco, P. W., Stark, K. P. (eds.) Proc. Inaug. Great Barrier Conf. J.C.U. Press, Townsville, p. 161-167

Borowitzka, M. A., Larkum, A. W. D. (1986). Reef algae. Oceanus 29: 49-54

Borowitzka, M. A., Larkum, A. W. D., Borowitzka, L. J. (1978). A preliminary study of algal turf communities of a shallow coral reef lagoon using an artificial substratum. Aquat. Bot. 5: 365-381

Bradbury, R. H., Loya, Y., Reichelt, R. E., Williams, W. T. (1986). Patterns in the structural typology of benthic communities on two coral reefs of the central Great Barrier Reef. Coral Reefs 4: 161-167

Brawley, S. H., Adey, W. H. (1977). Territorial behaviour of three spot damselfish (Eupomacentrus planifrons) increases reef algal biomass and productivity. Environ. Biol. Fish. 2: 45-51

Carpenter, R. C. (1985). Relationships between primary production and irradiance in coral reef algal communities. Limnol. Oceanogr. 30: 784-793

Carpenter, R. C. (1986). Partitioning herbivory and its effects on coral reef algal communities. Ecol. Monogr. 56: $345-363$

Dahl. A. L. (1974). The structure and dynamics of benthic algae in the coral reef ecosystem. Proc. 2nd. Int. Symp. Coral Reefs 1. 21-26

Done, T. J. (1982). Patterns in the distribution of coral communities across the central Great Barrier Reef. Coral Reefs 1: 95-- 107

Drew, E. A. (1983). Halimeda biomass, growth rates and sediment generation on reefs in the central Great Barrier Reef province. Coral Reefs 2: 101-110 
Hackney, J. M., Carpenter, R. C., Adey, W. H. (1989). Characteristic adaptations to grazing among algal turfs on a Caribbean coral reef. Phycologia 28: 109-119

Hatcher, B. G. (1982). The interaction between grazing organisms and the epilithic algal community of a coral reef: a quantitative assessment. In: Gomez, E. (ed.) Proc. 4th Int. Symp. Coral Reefs, Vol. 2. Univ. of the Philippines, Marine Sciences Center, Quezon City, p. 515-524

Hatcher, B. G. (1983). Grazing in coral reef ecosystems. In: Barnes, D. J. (ed.) Perspectives on coral reefs, B. Clouston, Manuka, A.C.T., p. 164-169

Hatcher, B. G., Larkum, A. W. D. (1983). An experimental analysis of factors controlling the standing crop of the epilithic algal community on a coral reef. J. exp. mar. Biol. Ecol. 69: 61-84

Hay, M. E. (1981). Spatial patterns of grazing intensity on a Caribbean barrier reef: herbivory and algal distribution. Aquat. Bot. 11: 97-109

Klumpp, D. W., McKinnon A. D. (1989). Temporal and spatial patterns in the primary production of a coral reef epilithic algal community. J. exp. mar. Biol. Ecol. 131: 1-22

Klumpp, D. W., McKinnon, D., Daniel, P. (1987). Damselfish territories: zones of high productivity on coral reefs. Mar. Ecol. Prog. Ser. 40: 41-51

Klumpp, D. W., Polunin, N. V. C. (1989). Partitioning of food resources between grazers within damselfish territories on a coral reef. J. exp. mar. Biol. Ecol. 125: 145-169

Klumpp, D. W., Polunin, N. V. C. (1990). Algal production, grazers and habitat partitioning on a coral reef: positive correlation between grazing rate and food availability. In: Barnes, M., Gibson, R. N. (eds.) Trophic relationships in the marine environment. Aberdeen Univ. Press, Aberdeen, p. 372-388

Littler, M. M., Arnold, K. E. (1982). Primary productivity of marine macroalgal functional-form groups from southwestern North America. J. Phycol. 18: 307-311

Marsh, J. A. (1976). Energetic role of algae in reef ecosystems. Micronesica 12: 13-21

Morrissey, J. (1980). Community structure and zonation of macroalgae and hermatypic corals on a fringing reef flat of

This article was submitted to the editor
Magnetic Island (Queensland, Australia). Aquat. Bot. 8: $91-139$

Mundy, C. N. (1990). Field and laboratory investigations of the line transect technique for monitoring the effects of the crown-of-thorns starfish, Acanthaster planci. The Crownof-Thorns Study. Australian Institute of Marine Science, Townsville

Odum, H. T., Odum, E. P. (1955). Trophic structure and productivity of a windward coral reef community on Eniwetok Atoll. Ecol. Monogr 25: 291-320

Rogers, C. S., Salesky, N. (1981). Productivity of Acropora palmata, macroscopic algae, and algal turfs from Tague Bay reef, St. Croix, U. S. Virgin Islands. J. exp. mar. Biol. Ecol. 49: 179-187

Russ, G. R. (1987). Is rate of removal of algae by grazers reduced inside territories of tropical damselfishes? J. exp. mar. Biol. Ecol. 110: 1-17

SAS (1985). SAS users guide: statistics. Version 5 edn. SAS Institute Inc., Cary, NC

Scott, F. J., Russ, G. R. (1987). Effects of grazing on species composition of the epilithic algal community on coral reefs of the central Great Barrier Reef. Mar. Ecol. Prog. Ser. 39 . 293-304

Steneck, R. S. (1985). Adaptations of crustose coralline algae to herbivory: patterns in space and time. In: Toomey, D. F., Nitecki, M. H. (eds.) Paleoalgology: contemporary research and applications. Springer-Verlag, Berlin, p. $352-366$

Wanders, J. B. W. (1976). The role of benthic algae in a shallow reef of Curaçao (Netherlands Antilles). I. Primary productivity in the coral reef. Aquat. Bot. 2: 235-270

Wanders, J. B. W. (1977). The role of benthic algae in the shallow reef of Curaçao (Netherlands Antilles). III: The significance of grazing. Aquat. Bot. 3: 357-390

Wilkinson, C. R. (1986). The nutritional spectrum in coral reef benthos - or sponging off one another for dinner. Oceanus 29: $68-73$

Williams, D. McB., Russ, G. R., Doherty, P. J. (1986). Reef fish: large scale distribution and recruitment. Oceanus 29: $76-82$

Manuscript first received: March 19, 1992

Revised version accepted: July 1, 1992 